\title{
Análise da mancha de inundação da Barragem Sul da Mina de Brucutu, São Gonçalo do Rio Abaixo, MG
}

\author{
Caio Moreira dos Santos ${ }^{1}$ \\ Junior Geraldo da Silva ${ }^{2}$ \\ Pedro Valle Salles ${ }^{3}$ \\ Flávia Cristina Silveira Braga ${ }^{4}$ \\ Flávia Spitale Jacques Poggiali ${ }^{5}$
}

\begin{abstract}
RESUMO
Nos últimos anos, o Brasil tem enfrentado desastres ambientais e sociais ocasionados por barragens de rejeito das mineradoras, em grande parte, decorrentes da falta de controle e da falta de fiscalização dessas estruturas. Destacam-se as catástrofes ocorridas no estado de Minas Gerais, nos municípios de Mariana (em 2015) e Brumadinho (em 2019), que geraram maior preocupação com a estabilidade desse tipo de estrutura. Nesse contexto, este estudo apresenta os dados de uma simulação de rompimento da Barragem Sul da Mina Brucutu, utilizando o software REC-HAS e o AutoCAD Civil 3D, a fim de compará-los com os dados fornecidos pelo Plano de Ação de Emergência Para Barragens de Mineração (PAEBM) da empresa responsável pela Mina Brucutu. Para a realização dos cálculos foi criado um mapa de inundação e exportados os dados da bacia analisada, para o HECRAS. As simulações de rompimento forneceram resultados aproximados aos que foram fornecidos pelo PAEBM. No entanto, o fato do tempo de formação da brecha de ruptura estar incluída no tempo de chegada da onda de ruptura no PAEBM, é muito preocupante, pois é difícil estimar o início exato de formação da brecha.
\end{abstract}

Palavras-chave: Barragens de Rejeito. Mancha de Inundação. HEC-RAS. Desastre Ambiental.

\footnotetext{
${ }^{1}$ Graduado em Engenharia Civil pela Faculdade DOCTUM, Unidade João Monlevade, MG, Brasil. E-mail: caio.geologia@yahoo.com.br.

${ }^{2}$ Graduado em Engenharia Civil pela Faculdade DOCTUM, Unidade João Monlevade, MG, Brasil. E-mail: jr.silva010@gmail.com.

${ }^{3}$ Graduado em Engenharia Civil pela Universidade Federal de Minas Gerais - UFMG. Mestre e Doutorando em Engenharia Civil pelo Centro Federal de Educação Tecnológica de Minas Gerais - CEFET. Atua na Construção Civil e também é professor e pesquisador da Universidade do Estado de Minas Gerais - UEMG, Unidade João Monlevade, MG, Brasil. E-mail: pedrovallesalles025@gmail.com.

${ }^{4}$ Graduada, Mestra e Doutora em Geologia pela Universidade Federal de Minas Gerais - UFMG. Desenvolve pesquisas na área de Geologia e também é professora da Universidade do Estado de Minas Gerais - UEMG, Unidade João Monlevade, MG, Brasil. E-mail: flavia.braga@uemg.br.

${ }^{5}$ Graduada em Engenharia Civil, Mestra em Construção Civil e Doutora em Engenharia Metalúrgica e de Minas pela Universidade Federal de Minas Gerais - UFMG. Atualmente é pesquisadora e professora do Centro Federal de Educação Tecnológica de Minas Gerais - CEFET, Belo Horizonte, MG, Brasil. E-mail: flaviaspitale@gmail.com.
} 


\title{
Analysis of flood area of the South Dam from Brucutu Mine, São Gonçalo do Rio Abaixo, MG
}

\begin{abstract}
In recent years, Brazil has faced environmental and social disasters caused by mining tailings dams, largely due to the lack of control and the lack of inspection of these structures. Noteworthy are the catastrophes that occurred in the state of Minas Gerais, in the municipalities of Mariana (in 2015) and Brumadinho (in 2019), which generated greater concern about the stability of this type of structure. In this context, this study presents data from a simulation of rupture of the South Dam of Brucutu Mine, using the REC-HAS software and AutoCAD Civil 3D, in order to compare them with the data provided by the Emergency Action Plan for Dams Mining (PAEBM) of the company responsible for Brucutu Mine. In order to carry out the calculations, a flood map was created and the data from the analyzed basin was exported to HEC-RAS. The breakout simulations provided approximate results to those provided by PAEBM. However, the fact that the time of formation of the rupture gap is included in the time of arrival of the rupture wave in the PAEBM, is very worrying, since it is difficult to estimate the exact beginning of the gap formation.
\end{abstract}

Keywords: Tailings Dams. Flood Area. HEC-RAS. Environmental Disaster.

Artigo recebido em: 14/09/2020

Aceito em: 10/12/2020 


\section{INTRODUÇÃO}

As barragens de rejeitos são estruturas utilizadas por mineradoras como forma de armazenamento dos resíduos extraídos no processo de beneficiamento do minério. Esses resíduos são considerados uma fração estéril após a separação mecânica e/ou química do minério bruto em concentrado e rejeito.

Essas estruturas são feitas de terra e exercem a função de armazenamento desse material estéril. Em seu interior, ocorre uma segregação onde a parte sólida decanta para o fundo e a água, por sua vez, fica na superfície, sendo drenada, tratada e reutilizada no processo de mineração.

No passado, tais resíduos eram dispostos de forma que gerassem o menor custo de logística possível. Essa forma de descartar os rejeitos aliada à falta de controle e de fiscalização resultou em vários desastres ao longo dos anos, causando danos ambientais e sociais. Consequentemente, isso gerou uma maior preocupação com a estabilidade dessas estruturas.

Os desastres causados recentemente pelo rompimento de barragens de rejeitos no estado de Minas Gerais, despertaram dúvidas quanto à segurança e quanto ao correto monitoramento dessas estruturas. Em Mariana - MG, foram lançados no meio ambiente cerca de 35 milhões de $\mathrm{m}^{3}$ de rejeitos, causando a morte de 17 pessoas, desabrigando outras 1.265 e gerando incontáveis impactos ambientais (FREITAS, 2019). A qualidade da água do Rio Doce foi comprometida, principalmente devido ao volume de sólidos em suspensão, além de elevados teores de alumínio, ferro, manganês e arsênio, observados no período imediato após o rompimento da barragem de Mariana (DIAS et al., 2018). Já em Brumadinho, o desastre deixou 270 vítimas ao todo, sendo 253 mortes confirmadas e 17 pessoas que seguem desaparecidas, além dos 12 milhões de $\mathrm{m}^{3}$ de rejeitos (FREITAS, 2019). Em Brumadinho, 297,28 ha de terras foram soterradas pelos rejeitos, e pelo menos 193 estruturas empresariais $(41 \%)$ e familiares $(59 \%)$ foram comprometidas (PEREIRA et al., 2019). Segundo Lacaz et al. (2017) os relatórios elaborados por órgãos oficiais tais como Comissão Extraordinária de Barragens da Assembleia Legislativa de Minas Gerais e Ministério do Trabalho e da Previdência Social, indicam a incapacidade do Estado em cumprir seu papel de agente e fiscalizador e controlador das barragens.

Assim, este estudo analisará o Plano de Ação de Emergência das Barragens de Mineração (PAEBM) da Barragem Sul, localizada na Mina do Brucutu em São Gonçalo do 
Rio Abaixo, MG. Essa análise é de suma importância para verificar se realmente a população terá tempo hábil de evacuação, caso ocorra um rompimento. Apresenta-se os dados de uma simulação de rompimento da Barragem Sul, utilizando o software REC-HAS e o AutoCAD Civil 3D. Foi realizada ainda a comparação dos dados obtidos neste trabalho com os fornecidos pelo PAEBM da empresa responsável pela estrutura.

\section{BARRAGENS DE REJEITO}

Segundo Soares (2010), o método mais usual de armazenamento de rejeito da mineração é através de seu lançamento em lagos de decantação, ou aterros hidráulicos, represados por barragens. Em decorrência do aumento da demanda por produtos minerais nos últimos anos, essas estruturas sofreram ampliações em suas dimensões, o que exige um estudo mais aprofundado das mesmas e mais atenção ao seu monitoramento, devido ao seu alto potencial de dano em caso de falhas.

Sabe-se do alto risco de rompimento dessas estruturas e não surpreende que o rompimento de barragens de mineração é algo recorrente em Minas Gerais. De acordo com Oliveira (2015), de 1996 a 2016 foram identificados oito desastres de grande porte em Minas, considerando o desastre de Brumadinho em janeiro de 2019 são nove, o que representa um desastre a cada 2,55 anos no estado. As causas das falhas que originaram esses desastres são variadas. De acordo com Azam e Li (2010), apenas 27,5\% dos rompimentos de barragem, entre 1910 e 2009, tiveram como causas questões climáticas. As outras decorreram de infiltração (20,4\%), defeitos na estrutura e na fundação da barragem $(15,6 \%)$, falhas de manutenção $(12,5 \%)$ e instabilidade da encosta e transbordamento $(10,7 \%)$.

\subsection{Métodos de alteamento}

Na mineração, diversas vezes é necessário o desenvolvimento de complementos, ou seja, um aumento da capacidade das barragens. Com isso, são desenvolvidos os alteamentos, que são classificados de acordo com os métodos construtivos, sendo eles: o método a jusante; método da linha de centro e; método a montante.

Quando planeja-se realizar o alteamento de uma barragem, realiza-se um estudo para definir qual método é mais adequado, de acordo com várias características do empreendimento, tais como: topografia, hidrologia, geologia, tipos e propriedades do subsolo, granulometria e concentração dos rejeitos, velocidade de deposição, variação da 
capacidade de armazenamento do reservatório com o aumento da altura (SOARES, 2010). Sabe-se que muitas vezes na prática, infelizmente, as empresas preferem o método mais barato ao mais seguro.

O método à jusante, adotado na barragem foco do estudo (TETRA TECH, 2016), é considerado o mais seguro, pois tem como estrutura uma forma que não depende diretamente do rejeito e sua base consiste em uma área de alteamento a jusante do dique de partida. Seu modo de desenvolvimento visa reduzir os riscos de liquefação (CAMPOS, 1986). Segundo Campos (1986), esse método possui menor probabilidade de ruptura interna, porém tem como desvantagem seu elevado custo construtivo, maior quantidade de aterro necessário para execução e também, dentre os três tipos, necessita de uma maior área para sua construção.

\subsection{Mapa de inundação}

Os Mapas de Inundação compõem um importante conjunto de ferramentas de controle de uma situação de emergência proveniente de um rompimento de barragens. Devem apresentar, além da área atingida pela inundação, os locais para onde as vítimas deverão ser levadas, assim como os alojamentos temporários que deverão abrigar os atingidos pela inundação (SILVA, 2016). Esses mapas funcionam como base para a mineradora planejar a evacuação de moradores, considerando todas as habitações, acessos, pontos de encontro e infraestruturas existentes à jusante da barragem. Esses mapas são gerados através de softwares, sendo o REC-HAS um dos mais utilizados, por ser gratuito e apresentar resultados condizentes com os dados obtidos em campo. Além disso, Lauriano (2009) recomenda o uso do HEC-RAS pela possibilidade de utilização do programa consorciado a plataformas de Sistemas de Informações Geográficas (SIG), o que possibilita um melhor detalhamento das seções transversais e facilita o pós-processamento dos resultados para a geração dos mapas de inundação.

O HEC-RAS é desenvolvido pelo Centro de Engenharia Hidrológica do Corpo de Engenheiros do Exército Norte Americano e é um software de distribuição gratuita, de alta precisão e que permite realizar simulações de escoamento em regimes permanente e não permanente, assim como análise de sedimentos e de qualidade da água (NEVES, 2018).

Tanto Xiong (2011), quanto Ackerman e Brunner (2016) deixam claro a importância da avaliação da ruptura de barragens com o uso da ferramenta HEC-RAS. Através do 
software é possível realizar simulações de rompimentos em barragens de rejeito e também de água. Para isso é necessário conhecer as características geométricas do barramento e seu reservatório, as características da bacia de inundação e do curso d'água.

Outro ponto importante para a simulação de rompimento é a definição da brecha, que nada mais é que o início do rompimento da estrutura. Os parâmetros geométricos da brecha são de suma importância no mapa de inundação, uma vez que exercem influência na vazão de pico e no tempo de formação da mesma. Considerando esse aspecto, o modelo de Froehlich apresenta uma largura média cerca de quatro vezes menor do que a largura média do modelo de Von Thun e Gillette e uma vazão de pico duas vezes maior (NEVES, 2018). Portanto, durante a definição do modelo a ser utilizado, deve-se analisar os dois casos e considerar o de pior cenário possível.

\subsection{Métodos de monitoramento}

Os instrumentos de monitoramento implantados em barragens de rejeitos têm como objetivo monitorar o desenvolvimento de deformações e de pressões intersticiais na estrutura, além de obter dados de deslocamento, tensão total, vazão e nível de água. Esses dados são obtidos através de leituras periódicas e são comparados aos respectivos valores máximo e mínimo de controle, especificados nos critérios de projetos (MACHADO, 2007). Além desse monitoramento por equipamentos, é muito importante realizar inspeções de campo periodicamente em busca de alterações na estrutura da barragem. Os instrumentos mais comuns de monitoramento são os piezômetros, os marcos topográficos, inclinômetros, deformímetros e estações meteorológicas, entre outros.

Durante o planejamento de uma barragem, deve-se considerar vários fatores que podem ocasionar a ruptura da mesma. Destaca-se o rompimento devido às enchentes, falhas no sistema de extravasão, colmatação no sistema de drenagem, erosão regressiva interna (pipping), atividades sísmicas, liquefação, deficiência de compactação durante sua construção, escorregamentos internos ao entorno do reservatório, recalques excessivos do aterro ou fundação, entre outros.

\section{LOCALIZAÇÃO DA ÁREA DE ESTUDO}

A Barragem Sul (Figura 1) está inserida no município de São Gonçalo do Rio Abaixo, Minas Gerais, na mina de ferro Brucutu pertencente ao Complexo Mariana - Brucutu, 
administrada pela Vale. $\mathrm{O}$ acesso à barragem pode ser realizado pela BR-381, no trevo com a MG-129, seguindo em direção à Mina Brucutu, em seguida acessada por via local, dentro da área de mina.

A Mina de Brucutu está localizada à montante do centro urbano de São Gonçalo do Rio Abaixo. Aos pés da barragem corre o Rio Santa Bárbara, pertencente à Bacia do Rio Doce, que em caso de rompimento, seria o caminho natural da lama (Figura 1).

Figura 1: Acesso à Barragem Sul da Mina Brucutu

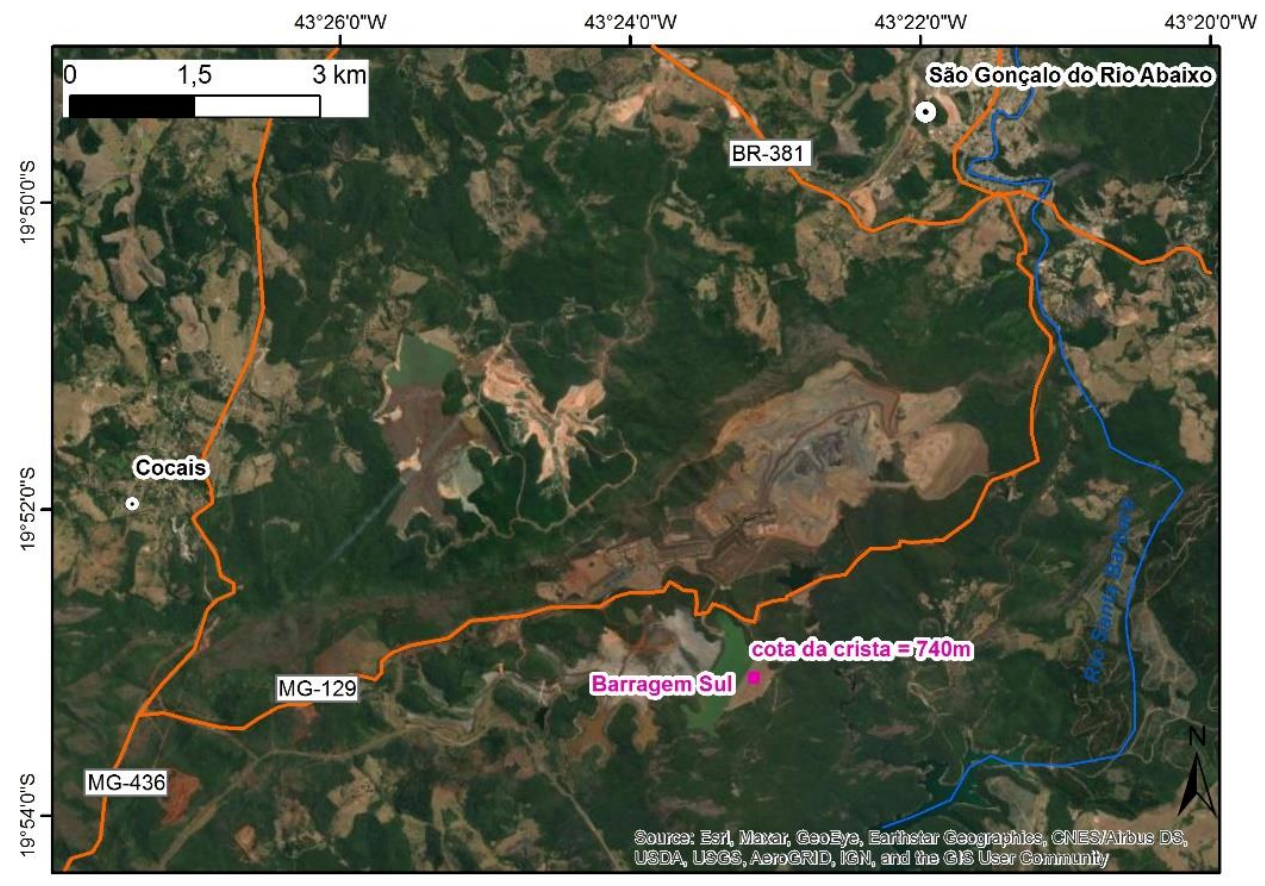

Fonte: Autoria própria.

A Barragem Sul foi construída a partir de um maciço inicial, seguido de alteamento a jusante e berma de reforço em aterro compactado. Sua drenagem interna é constituída por um sistema principal (formado por três tipos de filtro "sanduíche"), um sistema complementar (tapete drenante inclinado tipo sanduíche) e o sistema de conexão formado por duas linhas de poços drenantes (TETRA TECH, 2016).

Segundo o PAEBM, seu monitoramento é realizado a partir de piezômetros, indicadores de nível d'água, régua milimétrica, marcos superficiais e medidores de vazão. $\mathrm{O}$ vertedouro é constituído por um canal de aproximação, emboque de seção triangular com soleira espessa (em concreto), descida d'água em degraus, bacia de dissipação (em concreto) e canal de restituição (escavado em solo e revestido com enrocamento). São apresentados na Tabela 1 os principais dados da Barragem Sul de acordo com seu PAEBM elaborado pela 
Empresa TETRA TECH (2016).

Tabela 1: Dados gerais da Barragem Sul

\begin{tabular}{c|c}
\hline Informação & Valor \\
\hline Localização $(\mathrm{m})$ & 669.000 E e 7.800 .548 N (Sirgas 2000) \\
Cota da Crista $(\mathrm{m})$ & 740 \\
NA Normal $(\mathrm{m})$ & 837 \\
Altura da Barragem $(\mathrm{m})$ & 53.162 .357 \\
Volume do Reservatório $\left(\mathrm{m}^{3}\right)$ & 10.000 anos \\
Cheia de Projeto &
\end{tabular}

Fonte: TETRA TECH (2016).

\section{METODOLOGIA}

O mapeamento da mancha de inundação foi realizado com auxílio da ferramenta HECRAS inserida no AutoCAD 3D Civil. O primeiro passo foi a geração da superfície, ou modelo digital de terreno da bacia e o alinhamento representando o traçado do curso d'água onde ocorrerá a propagação da onda de inundação, proveniente do rompimento da barragem. Em seguida foram geradas seções transversais ao longo do curso d'água. Os objetos gerados foram exportados para o HEC-RAS para criação do modelo de inundação.

O modelo matemático utilizado para criação da mancha de inundação foi o de SaintVenant incorporado ao programa HEC-RAS, pois representa a metodologia mais conservadora e a que irá produzir um cenário de inundação mais catastrófico, possibilitando a preparação das autoridades a jusante para a pior situação (MELO et al., 2013). Para definição de áreas potencialmente inundáveis, utilizou-se a ferramenta River Analysis System do software HEC-RAS, a qual efetua cálculos de progressão bidimensional do escoamento.

A escolha pelo HEC-RAS (versão 5.0.7) se deu pela boa capacidade do modelo em representar os fenômenos de inundação de ruptura de barragens. Esse software é capaz de determinar as elevações de nível de água, tempo de chegada da onda de ruptura, velocidade máxima atingida na seção e outros resultados, baseando seus cálculos na solução das equações de Saint-Venant (WALM, 2018).

Mesmo que o AutoCAD Civil 3D forneça todas as informações necessárias para a simulação, ainda é necessário realizar alguns ajustes no HEC-RAS. Dessa forma, foram inseridas informações utilizadas durante os cálculos do rompimento, como hidrogramas, coeficiente de Manning e informações sobre o barramento. Todas as informações 
constituintes do modelo geométrico do HEC-RAS foram inseridas no modelo com auxílio da ferramenta SIG HEC-RAS (USACE, 2011) acoplada ao programa AutoCAD civil 3D.

O estudo da ruptura hipotética da Barragem Sul teve como objetivo o mapeamento das áreas potencialmente inundáveis da região à jusante do barramento. Neste contexto, considerou-se os dados hidrológicos fornecidos pelo PAEBM (Tabela 2). Posteriormente houve a definição do modo de falha e geração do hidrograma de ruptura, em seguida a propagação e mapeamento da mancha de inundação no vale a jusante do barramento.

\subsection{Superfície}

A superfície é o objeto do AutoCAD Civil 3D que representa a topografia do terreno através do modelo digital de terreno. É importante ressaltar que a topografia do terreno é um fator essencial na criação da geometria da simulação de rompimento de barragens.

Para a construção desse objeto, importou-se para o AutoCAD Civil 3D a imagem da área de estudo disponível no software Google Earth. Desta forma a imagem é inserida georreferrenciada no AutoCAD Civil 3D e o modelo digital do terreno é criado. A partir da definição da superfície de estudo, gerou-se uma nuvem de pontos georreferenciados e as curvas de nível.

Tabela 2: Síntese dos principais dados hidrológicos

\begin{tabular}{c|c}
\hline Descrição & Valor adotado \\
\hline Tempo de recorrência chuva de projeto (anos) & 10.000 \\
\hline Área de drenagem $\left(\mathrm{km}^{2}\right)$ & 11,9 \\
\hline Duração da chuva de projeto $(\mathrm{h})$ & 10 \\
\hline Altura da chuva de projeto $(\mathrm{mm})$ & 230 \\
\hline Nível da crista da barragem $(\mathrm{m})$ & 740,00 \\
\hline Nível de água normal do reservatório $(\mathrm{m})$ & 737,00 \\
\hline Nível de água máximo do reservatório $(\mathrm{m})$ & 738,39 \\
\hline Borda livre remanescente $(\mathrm{m})$ & 1,61 \\
\hline Vazão máxima afluente da área de drenagem $\left(\mathrm{m}^{3} / \mathrm{s}\right)$ & 125 \\
\hline Vazão máxima efluente do reservatório $\left(\mathrm{m}^{3} / \mathrm{s}\right)$ & 64,2 \\
\hline Volume do lago $\left(\mathrm{m}^{3}\right)$ & 4.248 .211 \\
\hline Volume disponível para o trânsito de cheias $\left(\mathrm{m}^{3}\right)$ & 2.551 .026 \\
\hline Volume total de sólidos depositados $\left(\mathrm{m}^{3}\right)$ & 48.914 .146 \\
\hline
\end{tabular}

Fonte: TETRA TECH (2016). 


\subsection{Alinhamento}

O alinhamento é responsável por definir o traçado do curso d'água onde ocorrerá a propagação da onda de inundação, proveniente do rompimento da barragem. Trata-se de um objeto bidimensional que, nesse caso, foi criado a partir do contorno do Rio Santa Bárbara, até o final da mancha urbana de São Gonçalo do Rio Abaixo. Apesar de ser um objeto bidimensional, é possível obter informações de sua interação com o terreno, e assim analisar de que forma o curso d'água interage com a planície de inundação. Esta interação é fundamental em um estudo de escoamento no HEC-RAS, já que características do terreno são fundamentais para calcular o nível da inundação.

\subsection{Seções Transversais}

A ferramenta utilizada para criar as seções transversais é a Sample Line. A Sample Line será responsável por fornecer ao HEC-RAS a interação entre o curso d'água e o terreno, delimitando assim o canal e suas margens.

Conforme ilustrado na Figura 2, é possível criar um conjunto de Sample Lines no Civil 3D, de acordo com as características da simulação que será realizada e pré-visualizada, de forma a analisar melhor as nuances do terreno que será utilizado para realização do estudo.

Figura 2: Superfície do terreno à jusante do barramento, o alinhamento do Rio Santa Bárbara (em rosa) e as seções transversais geradas para análise (em azul)

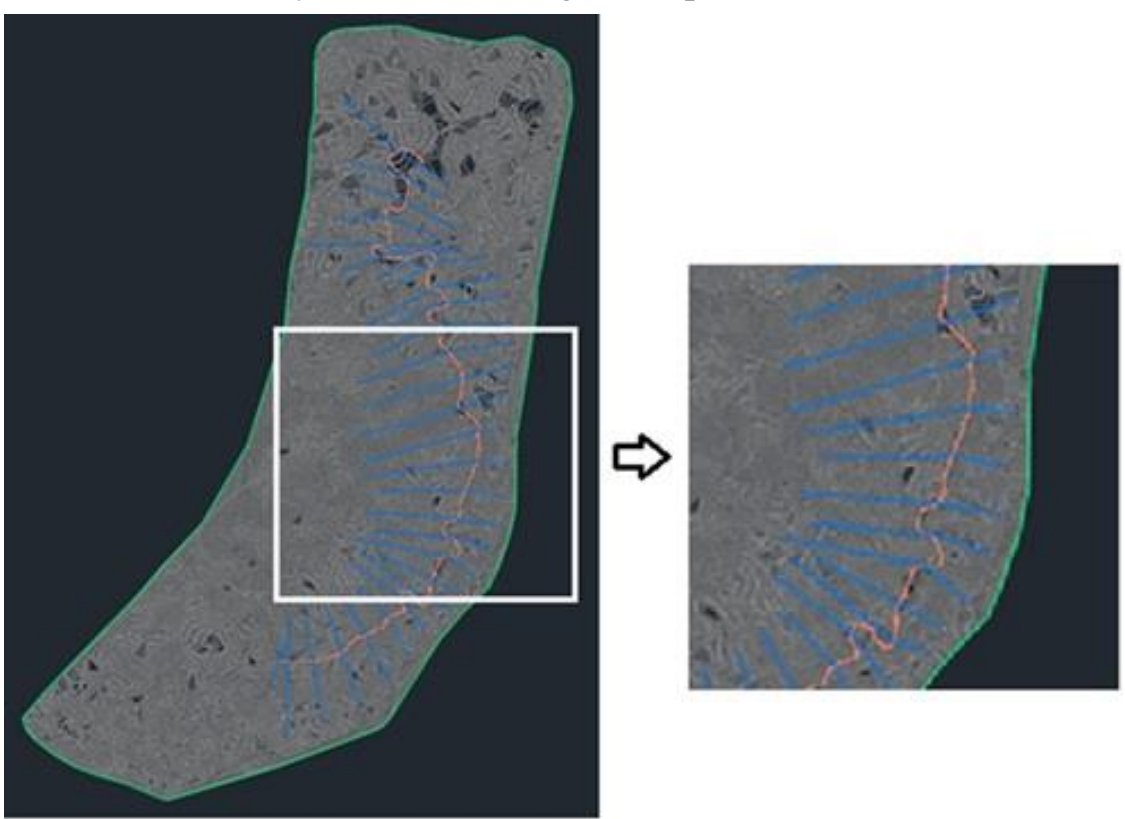

Fonte: Autoria própria. 


\subsection{Exportação para o HEC-RAS}

Os objetos anteriores são exportados para o HEC-RAS através de uma ferramenta presente no AutoCAD, que ajusta toda a geometria criada para o formato GEO, aceito pelo HEC. A modelagem no HEC-RAS consiste na configuração da geometria (seção da barragem e do reservatório), do regime não permanente e o plano de simulação do sistema. Em seguida, realiza-se no HEC-RAS a configuração da barragem com a brecha de ruptura de acordo com as informações presentes no PAEBM da Barragem Sul.

Como o objetivo deste trabalho é confirmar as informações contidas no PAEBM, os dados hidrográficos e parâmetros da barragem foram conservados. Sendo assim, de acordo com TETRA TECH (2016), a geometria final da brecha foi estimada a partir do modelo empírico de Froehlich (2008). O modelo da brecha tem formato de prisma trapezoidal com base maior e menor, respectivamente de $125,00 \mathrm{~m}$ e 20,00 m, altura de 75 metros e inclinação lateral de $1 \mathrm{~V}: 0,7 \mathrm{H}$. Entretanto, nessa simulação será adota a altura de 83 metros para a brecha. A elevação no nível de água para o desenvolvimento da brecha é de 738,39 m e o volume de água acima da cota de fundo da brecha é de $4.248 .211 \mathrm{~m}^{3}$. Quanto ao tempo de formação da brecha, adotou-se 22 minutos.

A partir da geomorfologia e das imagens de satélite, observou-se que a planície de inundação do Rio Santa Bárbara no trecho estudado é predominantemente formada por meandros com vegetação e pedras, de condições regulares. Sendo assim, adotou-se o valor de 0,040 para o Coeficiente de Manning, conforme presente na Tabela 3.

Durante a abertura da brecha o reservatório é drenado e as vazões constituintes do hidrograma efluente são calculadas considerando o estrangulamento do escoamento, a carga hidráulica a montante e as dimensões instantâneas da brecha. No âmbito desse trabalho, essa simulação foi realizada pela aplicação da lei de conservação da massa introduzida no modelo de armazenamento incorporado ao programa HEC-HAS (USACE, 2011).

Segundo Lucia (1981), na ausência de evidências contrárias, todo o volume armazenado no reservatório será considerado para o cálculo do hidrograma efluente. Além disso, recomenda-se utilizar a altura do barramento como altura final da brecha. Entretanto, nos cálculos da TETRA TECH (2016) considerou-se a altura final da brecha como $75 \mathrm{~m}$. 
Tabela 3: Síntese dos principais dados hidrológicos

\begin{tabular}{c|c|c|c|c|c}
\hline \multirow{2}{*}{ Item } & Natureza das paredes & \multicolumn{4}{|c}{ Condições } \\
& Limpos, retilíneos e uniformes. & 0,025 & 0,028 & 0,030 & 0,033 \\
\hline 1 & Como em 1, porém com vegetações e pedras. & 0,030 & 0,033 & 0,035 & 0,040 \\
2 & Com meandros, bancos e poços pouco profundos, & 0,035 & 0,040 & 0,045 & 0,050 \\
4 & Como em 3, águas baixas, declividade fraca. & 0,040 & 0,045 & 0,050 & 0,055 \\
5 & Como em 3, com vegetação e pedras. & 0,033 & 0,035 & 0,040 & 0,045 \\
6 & Como em 4, com pedras. & 0,045 & 0,050 & 0,055 & 0,060 \\
7 & Com margens espraiadas, pouca vegetação. & 0,050 & 0,060 & 0,070 & 0,080 \\
8 & Com margens espraiadas, muita vegetação. & 0,075 & 0,100 & 0,125 & 0,150 \\
\hline
\end{tabular}

Fonte: Porto (2006).

\section{RESULTADOS}

A modelagem no HEC-HAS tem como objetivo encontrar o hidrograma de vazão a jusante da Barragem Sul. Obteve-se uma mancha de inundação informando as áreas afetadas pelo possível rompimento da barragem e dados em formato de tabela com informações de seções pré-estabelecidas.

Após a simulação das manchas de inundação, o mapa foi extraído para o programa AutoCAD. Na Figuras 3 temos a comparação da mancha obtida neste trabalho com a fornecida pelo PAEBM.

A mancha gerada pelo presente trabalho atingiu uma área maior de inundação (Figura 3). Essa diferença pode ser explicada pela diferença entre as duas superfícies utilizadas para o estudo. Enquanto o estudo do PAEBM baseia-se em dados obtidos em campo, como na batimetria do Rio Santa Bárbara, o presente estudo baseia-se em altimetria gerada por imagens de satélite (Landsat 8) disponíveis no Google Earth, o que pode gerar resultados menos precisos, pois o satélite possui resolução de $15 \mathrm{~m}$ no pancromático e de $30 \mathrm{~m}$ no multiespectral (INPE, 2018). Outro fato que pode explicar essa diferença é a altura total utilizada para brecha de rompimento, $83 \mathrm{~m}$ neste estudo e $75 \mathrm{~m}$ no PAEBM. Apresenta-se a seguir as Tabelas 4 e 5, a primeira contendo informações presentes no PAEBM, e a segunda com os dados obtidos pelo presente estudo. 
Figura 3: Mancha de inundação fornecida pelo PAEBM (TETRA TECH, 2016) e a gerada pelo HEC-HAS neste trabalho

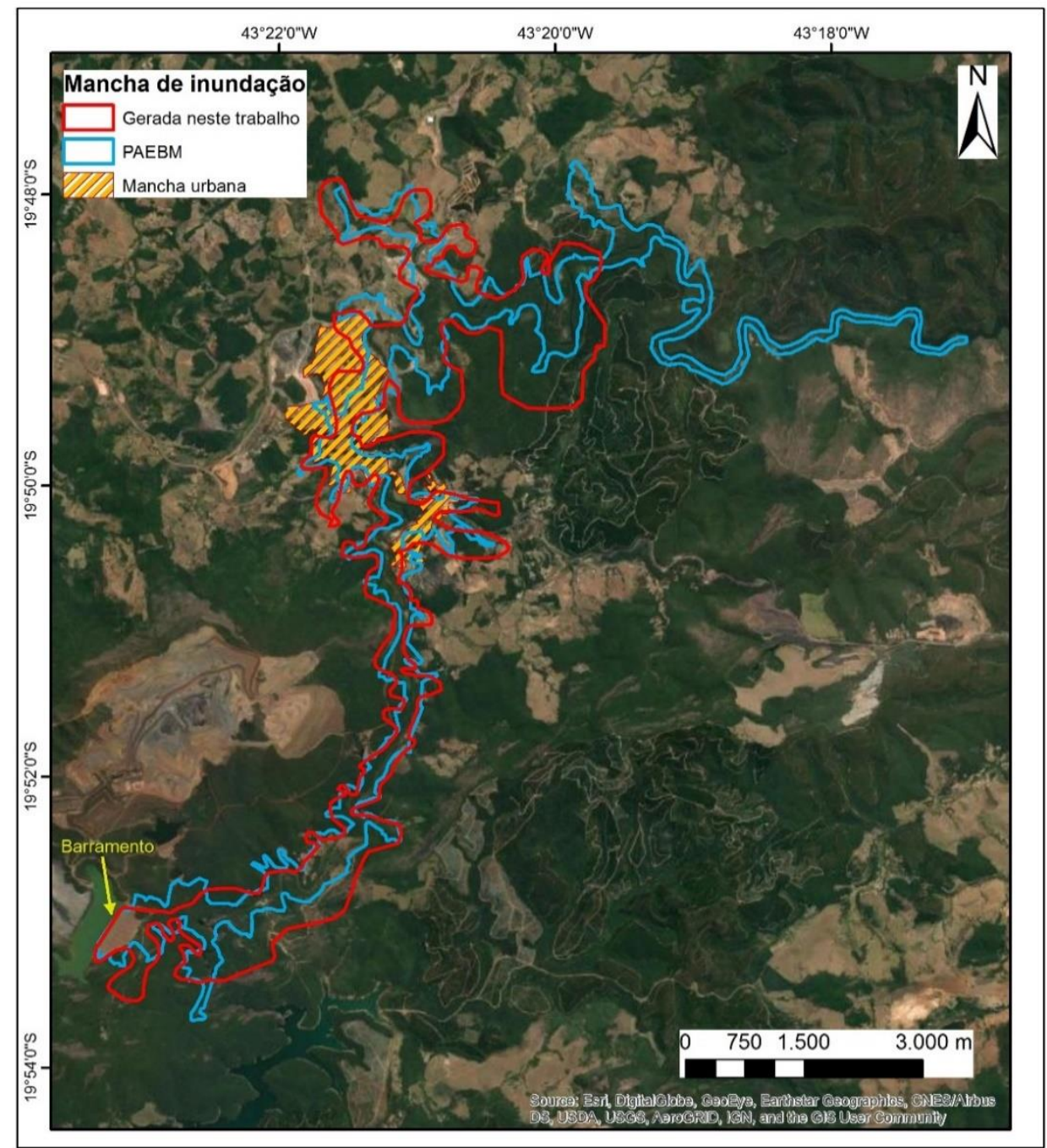

Fonte: Autoria própria.

\section{DISCUSSÕES}

De acordo com a Tabela 4, a simulação realizada pela empresa TETRA TECH (2016) se estendeu por $32 \mathrm{~km}$, com seções de quatro em quatro km e uma seção na ZAS (10 km à jusante do barramento). Em uma análise preliminar, percebe-se incoerências nas informações contidas no PAEBM, como na distância do eixo da barragem em relação à comunidade Vargem da Lua e o tempo de chegada da onda de ruptura à comunidade. 
Tabela 4: Síntese dos principais dados hidrológicos

\begin{tabular}{|c|c|c|c|c|c|c|c|}
\hline Descrição de referência & $\begin{array}{c}\text { Distância } \\
\text { em relação } \\
\text { ao eixo da } \\
\text { Barragem } \\
(\mathrm{km}) \\
\end{array}$ & $\begin{array}{l}\text { Profundidade } \\
\text { Máxima Atingida } \\
\text { na Seção }(\mathbf{m})\end{array}$ & $\begin{array}{c}\text { Elevação } \\
\text { Máxima } \\
\text { Atingida na } \\
\text { Seção (m) }\end{array}$ & $\begin{array}{l}\text { Velocidade } \\
\text { Máxima } \\
\text { Atingida na } \\
\text { Seção }(\mathrm{m} / \mathbf{s})\end{array}$ & $\begin{array}{c}\text { Tempo de } \\
\text { Chegada da } \\
\text { Onda de } \\
\text { Ruptura - } 2 \\
\text { Pés (hh:mm) } \\
\end{array}$ & $\begin{array}{c}\text { Tempo de } \\
\text { Chegada da } \\
\text { Onda de } \\
\text { Ruptura - Pico } \\
\text { (hh:mm) } \\
\end{array}$ & $\begin{array}{c}\text { Vazão Máxima } \\
\text { Atingida na } \\
\text { Seção (m³/s) }\end{array}$ \\
\hline Eixo da Barragem & 0 & $\mathrm{~N} / \mathrm{A}$ & $\mathrm{N} / \mathrm{A}$ & $\mathrm{N} / \mathrm{A}$ & $\mathrm{N} / \mathrm{A}$ & $\mathrm{N} / \mathrm{A}$ & $\mathrm{N} / \mathrm{A}$ \\
\hline $\begin{array}{l}\text { Comunidade de Vargem da } \\
\text { Lua }\end{array}$ & 4 & 29,8 & 662,5 & 3,11 & $00: 25$ & $00: 35$ & 10.874 \\
\hline $\begin{array}{l}\text { A Montante da Mancha } \\
\text { Urbana do Município de São } \\
\text { Gonçalo do Rio Abaixo }\end{array}$ & 8 & 20,4 & 647,5 & 4,84 & $00: 40$ & 01:00 & 5.325 \\
\hline $\begin{array}{l}\text { Zona de Autossalvamento } \\
\text { (ZAS) }\end{array}$ & 10 & 22,3 & 645,0 & 1,39 & $00: 50$ & 01:20 & 3.321 \\
\hline $\begin{array}{c}\text { Bairro Santa Efigênia (São } \\
\text { Gonçalo do Rio Abaixo) }\end{array}$ & 12 & 17,4 & 642,4 & 2,15 & 01:00 & $01: 40$ & 2.811 \\
\hline- & 16 & 15,8 & 637,5 & 1,67 & $01: 50$ & $03: 30$ & 1.567 \\
\hline- & 20 & 14,2 & 633,8 & 1,12 & $02: 40$ & $04: 10$ & 1.420 \\
\hline- & 24 & 12,2 & 629,3 & 1,23 & 03:05 & $05: 40$ & 1.354 \\
\hline $\begin{array}{c}\text { A Montante da Pequena } \\
\text { Central Hidrelétrica }(\mathrm{PCH}) \\
\text { São Gonçalo }\end{array}$ & 28 & 15,9 & 627,8 & 1,95 & $03: 55$ & $06: 25$ & 1.340 \\
\hline $\begin{array}{c}\text { A Jusante da Pequena Central } \\
\text { Hidrelétrica (PCH) São } \\
\text { Gonçalo }\end{array}$ & 32 & 19,8 & 600,3 & 3,55 & $04: 35$ & $07: 30$ & 2.680 \\
\hline
\end{tabular}

Fonte: TETRA TECH (2016). 
Tabela 5: Dados da simulação realizada através do programa HEC-HAS neste trabalho

\begin{tabular}{|c|c|c|c|c|c|c|c|}
\hline Descrição de referência & $\begin{array}{c}\text { Distância em } \\
\text { relação ao eixo } \\
\text { da Barragem } \\
(\mathbf{k m})\end{array}$ & $\begin{array}{l}\text { Profundidade } \\
\text { Máxima } \\
\text { Atingida na } \\
\text { Seção (m) }\end{array}$ & $\begin{array}{l}\text { Elevação } \\
\text { Máxima } \\
\text { Atingida na } \\
\text { Seção (m) }\end{array}$ & $\begin{array}{l}\text { Velocidade } \\
\text { Máxima } \\
\text { Atingida na } \\
\text { Seção }(\mathbf{m} / \mathbf{s})\end{array}$ & $\begin{array}{c}\text { Tempo de } \\
\text { Chegada da } \\
\text { Onda de } \\
\text { Ruptura - } 2 \\
\text { Pés (hh:mm) } \\
\end{array}$ & $\begin{array}{c}\text { Tempo de Chegada } \\
\text { da Onda de } \\
\text { Ruptura - Pico } \\
\text { (hh:mm) }\end{array}$ & $\begin{array}{c}\text { Vazão Máxima } \\
\text { Atingida na Seção } \\
\left(\mathrm{m}^{3} / \mathbf{s}\right)\end{array}$ \\
\hline Eixo da Barragem & 0 & N/A & $\mathrm{N} / \mathrm{A}$ & $\mathrm{N} / \mathrm{A}$ & $\mathrm{N} / \mathrm{A}$ & N/A & N/A \\
\hline $\begin{array}{l}\text { Comunidade de Vargem } \\
\text { da Lua }\end{array}$ & 3 & 31,8 & 664,5 & 6,73 & $00: 25$ & $00: 32$ & 14.243 \\
\hline Comunidade de Roque & 5 & 29,8 & 662,5 & 5,10 & $00: 27$ & $00: 35$ & 9.877 \\
\hline Bairro Gralhos & 7 & 25,3 & 655,0 & 4,99 & $00: 30$ & $00: 45$ & 6.342 \\
\hline Bairro Malaquias & 8 & 25,0 & 647,5 & 3,84 & $00: 45$ & 01:00 & 5.739 \\
\hline $\begin{array}{c}\text { Zona de } \\
\text { Autossalvamento (ZAS) }\end{array}$ & 10 & 21,8 & 644,0 & 1,39 & $00: 50$ & $01: 15$ & 4.321 \\
\hline $\begin{array}{c}\text { Centro do Município São } \\
\text { Gonçalo do Rio Abaixo } \\
\text { (SGRA) }\end{array}$ & 11 & 15,3 & 640,5 & 1,27 & $01: 10$ & 02:00 & 3.118 \\
\hline Bairro Guanabara & 12 & 15,0 & 642,4 & 2,15 & 01:20 & $02: 20$ & 2.984 \\
\hline $\begin{array}{l}\text { Parque de Exposições de } \\
\text { SGRA }\end{array}$ & 13 & 12,2 & 639,3 & 1,23 & $01: 35$ & $02: 45$ & 2.642 \\
\hline $\begin{array}{l}\text { Final da Mancha Urbana } \\
\text { do Município de SGRA }\end{array}$ & 14 & 10,6 & 637,5 & 1,17 & 01:50 & 03:30 & 1.977 \\
\hline
\end{tabular}

Fonte: Autoria própria. 
Como as seções da simulação da Tabela 4 são realizadas em intervalos de 4 km, o tempo de chegada da onda de inundação à comunidade de Vargem da Lua está mascarado. Isso porque a população dessa comunidade está situada à 2,7 km de distância da barragem, ou seja, a informação de tempo de chegada real da onda de ruptura aos moradores é omitida.

Pensando nessa situação, o presente estudo realizou seções em locais estratégicos, onde há aglomerados de moradores e limitou a simulação até $14 \mathrm{~km}$, ou seja, até o final da mancha urbana de São Gonçalo do Rio Abaixo, diferentemente do fornecido pelo PAEBM. Os resultados dessa simulação estão presentes na Tabela 5.

Comparando as duas tabelas, percebe-se que os valores de vazão, velocidade, profundidade máxima atingida nas seções e tempo de chegada da onda são bem próximos. Devido à modificação da altura total da brecha $(75 \mathrm{~m}$ para o PAEBM e $83 \mathrm{~m}$ para esta simulação), a simulação do presente estudo apresentou maiores valores de profundidade, elevação, velocidade e vazões máximas em relação ao PAEBM. Além disso, a diferença de precisão dos modelos digital de terreno adotados pode provocar esses valores ligeiramente superiores.

O tempo de chegada da onda de ruptura (onda de 2 pés e de pico máximo) foram menores nas seções analisadas. Um detalhe importante, que não é mencionado pelo PAEBM é que no tempo de chegada da onda de ruptura já está contabilizado o tempo de formação da brecha, ou seja, 22 minutos. Sendo assim, de acordo com o PAEBM, o tempo de chegada da onda de ruptura à comunidade de Vargem da Lua são de 25 minutos a partir do início do pipping que forma a brecha e não 25 minutos após o rompimento.

Segundo o PAEBM, a população localizada na ZAS é alertada quando a barragem atinge o nível 3 de emergência, ou seja, risco de ruptura iminente ou a ruptura está ocorrendo. Nesse momento a brecha de ruptura já se iniciou. Levando em conta essas informações, a demora para identificação do início da brecha pode ser fatal para os moradores mais próximos da estrutura.

A seção localizada à $12 \mathrm{~km}$ do eixo da barragem, apresentou em ambas simulações uma velocidade maior do que a seção à montante. Isso pode ser explicado pelo estrangulamento da calha do rio frente ao relevo. Em seguida o relevo se torna mais plano e a velocidade do fluxo continua reduzindo.

A mancha de inundação gerada neste trabalho cobre 59\% da área urbana do município, já a mancha do PAEBM cobre $43 \%$ da área urbana (Figura 3). 
De uma forma geral, os resultados obtidos se mostraram compatíveis aos dados fornecidos pelo PAEBM da Vale. Entretanto, é necessária uma melhor calibração dos dados da superfície para resultados mais fidedignos, uma vez que o modelo HEC-RAS é sensível aos dados de topografia e aos dados de coeficiente de rugosidade de Manning.

\section{CONCLUSÕES}

Os resultados obtidos pelo presente estudo mostraram que os dados fornecidos pelo PAEBM da Vale estão de acordo com as metodologias utilizadas atualmente. Entretanto, é necessário melhorar a forma com que os dados são apresentados à população. $\mathrm{O}$ fato do tempo de formação da brecha de ruptura estar incluído ao tempo de chegada da onda de ruptura é muito preocupante, pois é difícil estimar o início exato de formação da brecha. Quando identificado o problema, já podem ter se passados minutos. Além disso, casos de negligência e imperícia no monitoramento de anomalias podem ser fatais. O PAEBM exige que o monitoramento em campo deve ser feito no máximo quinzenalmente, o que não atende às necessidades reais dos moradores à jusante da barragem. Recomenda-se que o monitoramento de barragens de rejeito seja em tempo real, com uso de tecnologias avançadas, levando-se em conta o risco que elas oferecem.

Vale ressaltar que, esse estudo não obteve os dados referentes ao monitoramento da Barragem Sul. Faz-se necessário conhecer os métodos de monitoramento (visual e eletrônico) e sua frequência, para um melhor apuramento do risco real dessa estrutura para a Comunidade de Vargem da Lua, que é a primeira a ser afetada em um possível rompimento.

Para a obtenção de resultados mais próximos do real, seria necessário a calibração do modelo para rompimento de barragens de rejeito, uma vez que o software foi desenvolvido para barragens de água. Outro fato que melhoraria os resultados seria a realização de levantamentos topográficos para delimitação mais precisa da topografia, além da obtenção do coeficiente de rugosidade de cada trecho estudado.

\section{REFERÊNCIAS}

ACKERMAN, C.T.; BRUNNER, G.W. Dam failure analysis using HEC-RAS and HECGeoRAS. In: Proceedings of Third Federal Interagency Hydrologic Modeling Conference, Reno, Reno, 8p, 2006.

AZAM, S; LI, Q. Tailings Dam Failures: A Review of the Last One Hundred Years. Geotechnical News, p. 50-53, dez., 2010. 
CAMPOS, T. M. P. Geotecnia e Meio Ambiente: Relato do Estado da Arte. In: VIII COBRAMSEF. Porto Alegre, 1986.

DIAS, C.A.; COSTA, A.S.V.; GUEDES, G.R.; UMBELINO, G.J. de M.; SOUSA, L.G.; ALVES, J.H., SILVA, T.G.M. Impactos do rompimento da barragem de Mariana na qualidade da água do rio Doce. Revista Espinhaço, v. 7, n. 1, p.21-35, 2018.

FREITAS, R. G1. 'A gente está resgatando sonhos e histórias', diz bombeiro que atuou em Brumadinho. Portal G1. Atualizado em 25/10/2019. Disponível em: https://g1.globo.com/ mg/minas-gerais/noticia/2019/10/25/a-gente-esta-resgatando-sonhose-historias-diz-bombeiro-que-atuou-em-brumadinho.html. Acesso em: 15 de nov. de 2019.

FROEHLICH, D. C. Embankment dam breach parameters and their uncertainties. Journal of Hydraulic Engineering, v. 134, n. 12, p. 1708-1721, 2008.

INPE - INSTITUTO DE PESQUISAS ESPACIAIS. Geração de Imagens. Coordenação Geral de Observação da Terra. Landsat. Disponível em: https://www.dgi.inpe.br/documentacao/satelites/landsat. Acesso em: 14 de dez. de 2019.

LACAZ, F.A. de C.; PORTO, M. F. de S.; PINHEIRO, T.M.M. Tragédias brasileiras contemporâneas: o caso do rompimento da barragem de rejeitos de Fundão/Samarco. Revista Brasileira de Saúde Ocupacional, v. 42, n.9, p. 1-12, 2017.

LAURIANO, A. W. Estudo de ruptura da Barragem de Funil: comparação entre os modelos FLDWAV e HEC-RAS. Dissertação de M.Sc. em Saneamento, Meio Ambiente e Recursos Hídricos, Escola de Engenharia, Universidade Federal de Minas Gerais. Belo Horizonte: Brasil, 2009. $251 \mathrm{f}$.

LUCIA, P. Review of experiences with flow failures of tailings dams and waste impoundments. $\mathrm{PhD}$, University of California. Berkeley: Estados Unidos, 1981. $228 \mathrm{f}$.

MACHADO, W. G. F. Monitoramento de Barragens de Contenção de Rejeitos Da Mineração. Dissertação de M.Sc., Departamento de Engenharia de Minas e de Petróleo. Escola Politécnica da Universidade de São Paulo. São Paulo: Brasil, 2007. 155 f.

MELO, L. P. R. et al. Análise comparativa de metodologias de propagação de ondas para previsão dos impactos de inundação provenientes da ruptura hipotética da barragem Tico-Tico. In: XX Simpósio Brasileiro de Recursos Hídricos. Universidade Federal de Minas Gerais, Escola de Engenharia. Belo Horizonte, 2013. 183 f.

NEVES, L. P. Segurança de Barragens - Legislação Federal Brasileira em Segurança de Barragens Comentada 2018. Disponível em: http://www.anm.gov.br/. Acesso em: 20 de maio de 2019.

OLIVEIRA, N. Minas já sofreu com outros rompimentos de barragens. O Tempo. (05/11/2015), 2015. Disponível em: http://www.otempo.com.br/cidades/minas-j\%3\%A1sofreu-com-outros-rompimentos-de-barragens-1.1159501. Acesso em: 10 de maio de 2019.

PEREIRA, L.F.; CRUZ, G. de B.; GUIMARÃES, R.M.F. Impactos do rompimento da barragem de rejeitos de Brumadinho, Brasil: uma análise baseada nas mudanças de 
cobertura da terra. Journal of Environmental Analysis and Progress, v. 4, n. 2, p.122-129, 2019.

PORTO, R. de M. Hidráulica Básica. 4. ed. São Paulo: Rima Artes e Textos, 2006. 540p.

SILVA, L. R. C. M. O AutoCAD Civil 3D auxiliando em Estudos de Rompimento de Barragens. 2016. Disponível em: http://www.aprenderweb.com.br/. Acesso em: 18 de junho de 2019.

SOARES, L. Barragem de Rejeitos. In: LUZ, A. B. L.; SAMPAIO, J. A.; FRANÇA, S. C. A. Tratamento de minérios. 5. ed. Rio de Janeiro: CETEM/MCT. 2010. p. 829-896.

TETRA TECH. Plano de Ação de Emergência Para Barragem de Mineração (PAEBM). Barragem Sul. Mina Brucutu, Complexo Mariana-Brucutu. Relatório. São Gonçalo do Rio Abaixo, 2016. 63 p.

USACE - U. S. ARMY CORPS OF ENGINEERS. HEC-GeoRAS, GIS Tools for Support of HEC-RAS using ArcGIS. User's Manual. Davis: Hydrologic Engineering Center (HEC), 2011, 244p.

XIONG, Yi. A Dam Break Analysis Using HEC-RAS. Journal Of Water Resource And Protection. Mississipi, p. 370-379, 28 mar., 2011.

WALM. Relatório do Estudo de Ruptura Hipotética (Dam Break) da Barragem 2. Disposição de Rejeitos e Estéril. SAM - Sul Americana de Metais S/A (Projeto Ferro Vale do Rio Pardo). 2018. Disponível em: http://ftp.brandt.com.br:2100/EIA\%20SAM\%20BLOCO\%208/EIA\%20SAM\%20BLOCO 8\%20-\%20PASTAS\%20DE\%20ARQUIVOS/EIA\%20$\% 20 \mathrm{CAP} \% \mathrm{C} 3 \% 8 \mathrm{DTULO} \% 2004 \% 20-$ $\% 20 \mathrm{Descri} \% \mathrm{C} 3 \% \mathrm{~A} 7 \% \mathrm{C} 3 \% \mathrm{~A} 3 \mathrm{o} \% 20 \mathrm{do} \% 20$ Empreedimento/ANEXOS/ANEXO\%208$\% 20 \mathrm{Dam} \% 20 \mathrm{Break} \% 20 \mathrm{da} \% 20 \mathrm{Barragem} \% 202 / \mathrm{ANEXO} \% 208 \% 20-\% 20 \mathrm{MINA} B \mathrm{BLC} 8007-$ 1010-G-RE-14_R03.pdf. Acesso em: 10 de maio de 2019. 